\title{
Global Impact: Elucidating Plant Responses to Viral Infection
}

\author{
Steven A. Whitham, ${ }^{1}$ Chunling Yang, ${ }^{1}$ and Michael M. Goodin ${ }^{2}$ \\ ${ }^{1}$ Department of Plant Pathology, lowa State University, Ames, IA 50011-1020, U.S.A.; ${ }^{2}$ Department of Plant Pathology, \\ University of Kentucky, Lexington, KY 40546, U.S.A.
}

Submitted 21 March 2006. Accepted 1 July 2006.

\begin{abstract}
Viruses induce a variety of responses in host cells that are mediated by perturbation of different signaling pathways. Advances in our understanding of the functions of viral proteins, plant biology in general, as well as technologies for profiling gene expression have converged in recent years to provide new insight into the events occurring inside susceptible and resistant host cells in response to virus infection. These effects range from nonspecific changes in gene expression due to the general accumulation of viral proteins to those responses that are initiated by the specific interactions between virus and host proteins. Here, we discuss a variety of expression profiling methods and approaches that have been used to study the effects of viruses on host transcriptomes. These studies have identified distinct sets of genes that have altered expression profiles in response to viruses, including stress- and defense-related genes. The activities of viral RNA silencing suppressors and interference with hormone signaling or biogenesis also influence plant gene expression and lead to developmental abnormalities.
\end{abstract}

Additional keyword: microarray, plant-virus interactions.

The obligate intracellular nature of viruses provides numerous opportunities for viral proteins and nucleic acids to interact with and influence the activity of host proteins and nucleic acids. As viral proteins and nucleic acids accumulate, they perform their various roles in replication, movement, encapsidation, and suppression of host defenses. In compatible interactions that result in systemic infections, each protein may have multiple functions that enable invasion throughout the plant and, ultimately, transmission to a new host. Frequently, viral proteins accumulate to extreme levels relative to host proteins, which is indicative of their proficiency in hijacking host cellular functions and resources. Accumulation of viral proteins and the accompanying infection is commonly associated with numerous effects in host cells as well as eliciting defects in plant growth and development. However, it is becoming increasingly evident that increases in the intracellular concentrations of viral proteins have many consequences for host gene expression and metabolism. Some of these effects do not necessarily provide an advantage to the virus but nevertheless have effects on the host. Thus, the distinction between virus-beneficial and the unintended consequences of infection are of interest to virologists and plant biologists alike.

Corresponding author: S. A. Whitham; 351 Bessey Hall; Telephone: +1.515.204.4952; Fax: +1.515.294.9420; E-mail: swhitham@ iastate.edu
One consequence of viral infection is the altered expression of host genes, which may have several root causes. A major challenge has been to identify host genes with altered mRNA transcription profiles and to decipher how and why the changes are initiated. The ultimate goal is to then use this information to investigate the functions of genes with altered expression profiles in plant-virus interactions. The different groups of coregulated genes lead to new hypotheses on how host cells are manipulated to create a favorable environment for infection, what defense and stress mechanisms are deployed, or how developmental defects resulting in symptoms are brought about (Fig. 1).

In this review, we discuss the techniques and experimental approaches that have been used to study host gene expression (Table 1), functional groups of genes whose expression profiles are altered in response to viral infection, and roles of viral RNA and protein components in eliciting changes in host gene expression (Fig. 1).

\section{Techniques and approaches}

for profiling gene expression in virus-host interactions.

Over the past decade, a variety of techniques have been used to identify the host gene-expression changes that occur as a result of virus or viroid infection. These techniques range from in situ hybridization with individual genes to global profiling of host mRNA transcripts using oligonucleotide or cDNA microarrays (Table 1). In situ hybridization has been performed on virus-infected embryonic tissues and cotyledons. This technique provides exquisite spatial resolution of the relationship between virus infection and the expression of a selected set of host genes but, with respect to viruses, has not been utilized to examine gene expression on a genome-wide scale. Methods for assaying host gene expression that do not rely on preselected genes for analysis have also been applied to plant-virus interactions. The application of these techniques has lacked the spatial resolution afforded by in situ hybridization but has permitted temporal changes in gene expression to be identified. These approaches, such as differential display and DNA macroor microarrays, each possess their own inherent advantages and disadvantages (Zhu et al. 2001).

To date, microarray-based studies of virus-host interactions have utilized the model dicot hosts Arabidopsis and Nicotiana benthamiana and have involved intact viruses or individual viral components (Table 1). While Arabidopsis experiments have used arrays derived from sequences of Arabidopsis thaliana origin, the $N$. benthamiana experiments utilized a heterologous array from Solanum tuberosum (potato), a related member of the Solanaceae. Heterologous microarrays, i.e., microarrays 
containing oligonucleotide or cDNA sequences from plants other than that used to provide the RNA for transcriptional profiling have not been used widely to investigate plant-pathogen interactions. The use of potato cDNA arrays for heterolo-

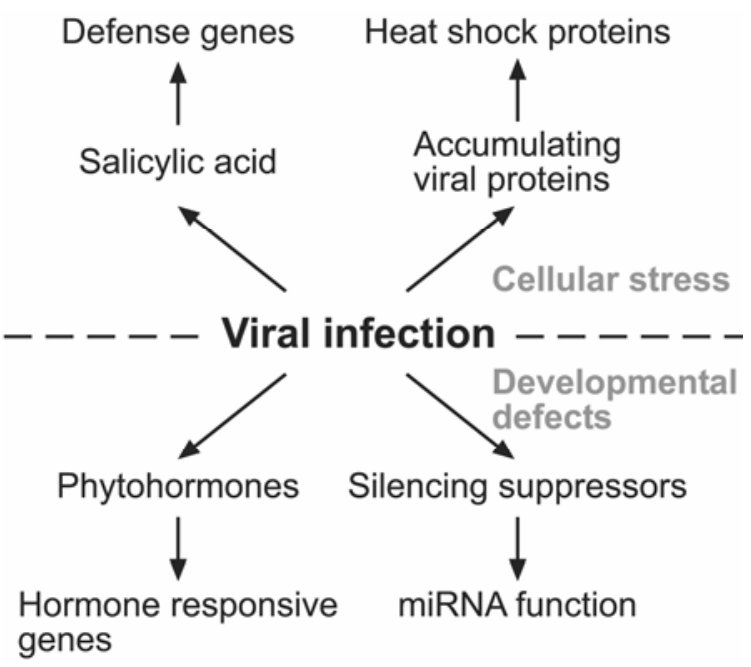

Fig. 1. Host responses and altered gene expression associated with plant virus infections. Compatible interactions between susceptible hosts and virulent pathogens frequently lead to the expression of suites of defense-related and heat shock genes. The expression of these genes is controlled by signaling pathways in the plants associated with initiation of defense responses and by other cellular stress response pathways. Viral infections can also disrupt the functions of regulatory small RNAs, such as micro RNAs, and phytohormone signaling or biosynthesis leading to developmental defects. gous studies is justified by recent analyses by Rensink and colleagues (2005), who analyzed 449,224 expressed sequence tags (EST) from six solanaceous species (potato, tomato, pepper, petunia, tobacco, and $N$. benthamiana). Assignment of these sequences into gene ontologies revealed that the transcripts within the gene indices encoded largely equivalent sets of biological functions. Furthermore, despite being derived from different tissues, 51 to $81 \%$ of the EST sequences had significant similarity at the nucleotide level with sequences among the six species. Such high degree of sequence conservation was supported by expression profiling using heterologous hybridization to potato cDNA arrays that contained 12,000 cDNA sequences and RNA for profiling derived from leaf tissue from each of the six species. Only 16 to $19 \%$ of the transcripts within the six gene indices did not have matches among Solanaceae, Arabidopsis, rice, or 21 other plant gene indices. These data prompted us to hypothesize the existence of a "core transcriptome" shared by the members of the Solanaceae. Therefore, high-density solanaceous arrays (SOLAR) with probes derived primarily from publicly available EST sequences from $N$. benthamiana, $N$. tabacum, $S$. tuberosum, and S. lycopersicon could provide near-genome coverage for a wide range of solanaceous species.

That a heterologous array could be used from studies of host-virus interactions was effectively demonstrated by Senthil and associates (2005), who successfully used arrays containing 15,000 cDNA sequences from $S$. tuberosum for identifying changes in plant gene expression in response to infection by the enveloped viruses, Sonchus yellow net nucleorhabdovirus (SYNV) and Impatiens necrotic spot tospovirus (INSV). Statistically significant changes $(P<0.01)$ in the expression of

Table 1. Selected studies employing different methods to profile host gene expression in response to viral infection.

\begin{tabular}{|c|c|c|c|c|c|}
\hline$\overline{\text { Host }}$ & Virus $^{a}$ & Focus of study & Tissue & Approach $^{b}$ & Reference \\
\hline \multicolumn{6}{|c|}{ In situ hybridization } \\
\hline Pea & PSbMV & Downregulated genes & Embryonic & 5 & Wang and Maule 1995 \\
\hline Pea & PSbMV & HSP70/ Ubiquitin & Embryonic & 5 & Aranda et al. 1996 \\
\hline Pea & $\begin{array}{l}\text { PSBMV, PEBV, } \\
\text { WCIMV, BCTV }\end{array}$ & HSP70/ lipoxygenase & Embryonic & 5 & Escaler et al. 2000b \\
\hline Сисurbita реро & CMV & HSP70/ NADP-ME & Cotyledons & 5 & Havelda and Maule 2000 \\
\hline \multicolumn{6}{|c|}{ Differential display } \\
\hline Arabidopsis & CaMV, CaMV gene VI & Global profiling & Systemic leaves & 1,4 & Geri et al. 1999 \\
\hline Chenopodium & TMV & Global profiling & Inoculated leaves & 2 & Cooper 2001 \\
\hline \multicolumn{6}{|c|}{ Serial analysis of gene expression (SAGE) } \\
\hline Cassava & ACMV & Global profiling & Mixed leaves & 1 & Fregene et al. 2004 \\
\hline \multicolumn{6}{|l|}{ Macroarray } \\
\hline Tomato & $\begin{array}{l}\text { PSTVd, TMV } \\
\text { Preselected by differential display }\end{array}$ & Global profiling & Systemic leaves & 1,2 & Itaya et al. 2002 \\
\hline Arabidopsis & CMV & Global profiling & Inoculated leaves & 1 & Ishihara et al. 2004 \\
\hline \multicolumn{6}{|l|}{ Microarray } \\
\hline Arabidopsis & $\begin{array}{l}\text { CMV, CaMV, ORMV } \\
\text { PVX, TVCV, TuMV }\end{array}$ & Transcription factors & Inoculated leaves & 1,2 & Chen et al. 2002 \\
\hline Arabidopsis & $\begin{array}{l}\text { CMV, ORMV, PVX } \\
\text { TVCV, TuMV }\end{array}$ & Global profiling & Inoculated leaves & 1,2 & Whitham et al. 2003 \\
\hline Arabidopsis & TMV & Global profiling & Systemic leaves & 1 & Golem and Culver 2003 \\
\hline Arabidopsis & CMV & Global profiling & Inoculated leaves & 1 & Marathe et al. 2004 \\
\hline Arabidopsis & CMV, ORMV & 388 Preselected genes & Inoculated leaves & 2,3 & Huang et al. 2005 \\
\hline$N$. benthamiana & INSV, SYNV & Global profiling & Systemic leaves & 1,2 & Senthil et al. 2005 \\
\hline $\begin{array}{l}\text { Arabidopsis } \\
\text { Proteomics }\end{array}$ & AC2 protein of MYMV & Global profiling & Protoplasts & 4 & Trinks et al. 2005 \\
\hline Rice & RYMV & Global profiling & Cell suspension & 1,3 & Ventelon-Debout et al. 2004 \\
\hline
\end{tabular}

${ }^{a}$ PSbMV = Pea seedborne mosaic virus $;$ PEBV = Pea early browning virus; WClMV = White clover mosaic virus; $\mathrm{BCTV}=$ Beet curly top virus; $\mathrm{CMV}=$ Cucumber mosaic virus; CaMV = Cauliflower mosaic virus; TMV = Tobacco mosaic virus; $\mathrm{ACMV}=$ African cassava mosaic virus; PSTVd = Potato spindle tuber viroid; ORMV = Oilseed rape mosaic virus; $\mathrm{PVX}=$ Potato virus $X$; TVCV = Turnip vein clearing virus; TuMV = Turnip mosaic virus; INSV = Impatiens necrotic spot tospovirus; $\mathrm{SYNV}=$ Sonchus yellow net nucleorhabdovirus; $\mathrm{MYMV}=$ Mung bean yellow mosaic virus; $\mathrm{RYMV}=$ Rice yellow mottle virus.

${ }^{\mathrm{b}}$ Experimental approach that the study is consistent with. $1=$ Profiling host responses to wild type or engineered viruses; $2=$ comparative analysis of host responses to different viruses, pathogens, and abiotic stresses; $3=$ host mutants or genotypes; $4=$ expression of individual virus proteins and nucleic acids; $5=$ Spatial and temporal relationships between viral infection and host responses. 
$275,2,646$, and 4,165 genes were detected in response to INSV at 2, 4, and 5 days postinoculation (dpi), respectively. For SYNV infection, significant changes in expression of 35 , 665 , and 1,458 genes at 5, 11, and $14 \mathrm{dpi}$, respectively, were identified. Perhaps related to the effect of the nuclear-replicating SYNV on host transcription, the fold changes in expression of commonly induced or repressed genes were always smaller in response to SYNV compared with the response to INSV, which replicates in the cytoplasm of infected cells. As noted for plus-strand RNA viruses, both virus-specific and common changes in gene expression were found, thereby contributing to a broader perspective to our understanding of virushost interactions. There are many economically important virushost interactions for which it may not be feasible to construct and use a microarray for a specific host. The pioneering work of Senthil and associates (2005) demonstrates that a common microarray can be used for studies with both model and agronomically important plants, thus facilitating the translation from basic to applied research.

The studies listed in Table 1 represent not only diverse expression profiling techniques but also a variety of experimental approaches designed to answer certain questions about either the effects of viruses, their individual components on a host, or both. We have classified each of the studies according to a type of experimental approach. The experimental classification scheme was adapted, in part, from previous studies (Jenner and Young 2005; Korth et al. 2005) that outlined frameworks to describe different types of studies that utilize microarrays to dissect the responses of mammalian host cells to viruses and other pathogens, regardless of the transcriptional profiling technology used. When taken together, the five experimental classes provide comprehensive information on the host genes that are modulated by viruses in space and time, the specific viral proteins involved, and the host signaling pathways that are perturbed.

Profiling host responses to wild-type or engineered viruses. Expression profiling of plant responses to wild-type viruses provides a baseline for understanding how different kinds of viruses affect host gene expression. Engineered viruses that differ in virulence from wild type can be utilized to study cellular responses to specific virus strains and provide insight into the functions of individual virus proteins.

Comparative analysis of host responses to different viruses, pathogens, and abiotic stresses. This approach may compare the host responses to diverse viruses with one another with the goal of discovering common responses or those specific to a particular virus. On a more grandiose scale, it may be useful to compare expression data generated from viral infections of plants with that of different pathogens or stresses. The goal of broad comparisons would be to reveal common responses to diverse biotic and abiotic stresses as well as specific responses to particular stresses.

Host mutants or genotypes. Analysis of different plant mutants and genotypes is useful for determining how host responses are controlled and whether they have effects on viral pathogenicity.

Expression of individual virus proteins and nucleic acids. The goal of this approach is to express individual virus components under control of constitutive or inducible promoters to study their effects on gene expression in different host genotypes, cell types, or tissues. Expression of individual or combinations of viral proteins will help to assign responses of coregulated genes to a specific viral protein or complex.

Spatial and temporal relationships between viral infection and host responses. Gene expression assays performed on samples of whole leaves or other tissues, while practical, cannot provide an accurate representation of the actual transcriptional changes that occur as a viral infection progresses. As shown in Table 1, several studies have used in situ hybridization to monitor changes in the expression of selected genes as viral infections progress (Maule et al. 2002). The experiments illustrate the principle that viral infection is a continuous and progressive process that occurs as a virus spreads across a tissue from a point source. Along with virus replication and movement, concomitant and conserved changes in host gene expression occur in embryonic and cotyledon tissues, such as increased expression of HSP70 and decreased expression of an assortment of other genes.

\section{Common responses of plants to viruses: \\ Cellular stress responses.}

Figure 1 outlines some common ways that a broad range of viruses may alter plant gene expression, and we expect that additional responses will be identified. The responses have been divided into two major categories of cellular stress and developmental defects. While these categories may not necessarily be exclusive, this division is convenient for purposes of discussing the individual responses, the associated gene expression changes, and the viral factors that initiate or contribute to the responses.

Comparison of Arabidopsis and N. benthamiana gene expression leads to the conclusion that virus infection causes characteristic changes in gene expression that resemble stress and defense responses (Fig. 1; Table 2). The stresslike responses are characterized by the induction of heat shock proteins (HSP) and defenselike responses by the induction of pathogenesisrelated (PR) genes and other genes associated with plant disease defense. We consider induction of HSP and PR genes to represent cellular stress responses because of their generic nature and the lack of specific elicitors that induce them. As shown below, it seems that most viruses trigger these generic responses, which occur in the absence of typical gene-for-gene or resistance gene-avirulence gene interactions.

Defense-related genes. The defense-related genes include numerous pathogenesis-related $(P R)$ genes such as $P R-1, P R-2$ ( $\beta-1,3$ glucanase), $P R-3$ (chitinase), $P R-4, P R-5$ (thaumatinlike protein), genes associated with redox status such as superoxide dismutase and GST (glutathione $S$-transferases), resistance gene homologs, and other genes of unknown function that are coinduced with the $P R$ genes (Table 2). WRKY6, a member of the $W R K Y$ family of transcription factors, is also induced in both Arabidopsis and $N$. benthamiana in response to viral infection (Table 2). This observation is interesting, given the ability of WRKYG to be induced by other pathogen infections and to regulate the expression of defense genes, such as $P R-1$ (Robatzek and Somssich 2001, 2002).

Incompatible interactions between viruses and hosts have previously served as models for investigating host defense responses. Many incompatible interactions are associated with increased $P R$ gene expression and protein accumulation mediated by salicylic acid (SA) (Gaffney et al. 1993; Malamy et al. 1990; Ryals et al. 1996). In general, increased production of $\mathrm{SA}$ is required for the dramatic accumulation of $P R$ mRNA transcripts and proteins that occurs during resistance response to viruses but not in susceptible interactions. Unlike those in incompatible interactions, SA levels are not altered in compatible virus-host interactions (Dempsey et al. 1997; Malamy et al. 1992), and thus, the amplification of a signal needed for robust $P R$ gene expression does not occur. Nevertheless, basal SA levels mediate the induction of defense-related genes in compatible host-virus interactions. The induction of $P R$ and other coregulated genes in response to Cucumber mosaic virus strain $Y(\mathrm{CMV}-\mathrm{Y})$ and Oilseed rape mosaic virus (ORMV) infection does not occur in $n a h G$ transgenic or sid2 plants that cannot 
accumulate SA (Huang et al. 2005). Cauliflower mosaic virus $(\mathrm{CaMV})$ also induces the expression of $P R-1$ in a SA-dependent manner during compatible interactions in Arabidopsis (Laird et al. 2004). The induction of $P R-1$ by CMV and ORMV was absolutely dependent on NPRl in addition to SA. In contrast, increased expression of $B G L 2, P R-5$, and most other defense-related genes tested did not require $N P R 1$, suggesting the pathway controlling expression of defense-related genes branches after SA (Fig. 2; Huang et al. 2005). Interestingly, $n a h G$ plants did not have enhanced susceptibility to ORMV, CMV, or CaMV, indicating that the defenselike response that is induced during infection has very little effect on these wild-type viruses (Huang et al. 2005; Laird et al. 2004). This observation is in contrast to the necessity of SA in resistance gene-mediated defense responses to CMV and Turnip crinkle virus (TCV) in Arabidopsis (Kachroo et al. 2000; Takahashi et al. 2002) or to the function of $N$-mediated resistance to Tobacco mosaic virus (TMV) in tobacco (Gaffney et al. 1993).

In compatible tobacco plants, CMV induces the expression of defense-related genes such as $P R-1$ and $P R-4 a$ (Ji and Ding 2001). As in Arabidopsis, the expression of these defenserelated tobacco genes was abrogated in $n a h G$ transgenic plants. Thus, a SA-dependent signaling pathway is also modulated by compatible viruses in tobacco. The $n a h G$ plants were more susceptible to the CMV $\Delta 2 \mathrm{~b}$ virus (lacking the $\mathrm{Cmv} 2 \mathrm{~b}$ protein) than wild-type plants suggesting, that loss of SA partially complemented the $2 \mathrm{~b}$ defect. In addition, recombinant TMV expressing Cmv2b accumulated to higher levels than wild-type TMV after SA application. Further analyses demonstrated that Cmv2b was antagonistic to the SA-mediated ex-

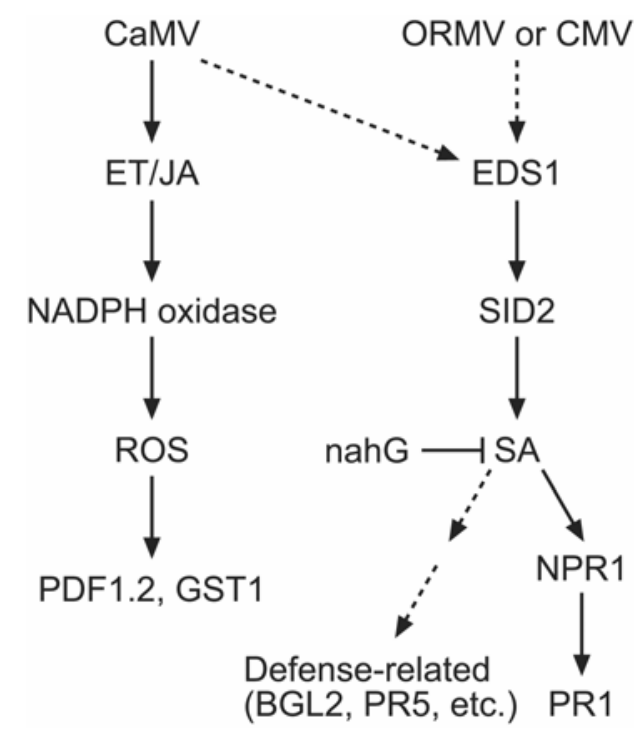

Fig. 2. Defenselike responses that are elicited by viruses in susceptible Arabidopsis plants. Cauliflower mosaic virus (CaMV) elicits the expression of jasmonic acid (JA)- and ethylene (ET)-responsive defense-related genes (PDF1.2 and GST1) as well as salicylic acid (SA)-dependent expression of pathogenesis-related genes $(P R-1)$. The dashed lines indicate that it is not known if SID2 or EDS1 are required to initiate the SAdependent gene expression in CaMV infections. Oilseed rape mosaic virus (ORMV) and Cucumber mosaic virus (CMV) elicit the expression of defense-related genes through a pathway dependent on EDS1, SID2, and SA. The expression of $P R-1$ and other defense-related genes $(B G L 2$ and $P R-5)$ appear to branch after SA, because increased $P R-1$ expression is strongly dependent on NPR1.

Table 2. Genes with common functions that are induced by positive- and negative-strand RNA viruses in Arabidopsis and Nicotiana benthamiana

\begin{tabular}{|c|c|c|c|}
\hline Description & Arabidopsis AGI number & Solanm tuberosum GenBank number ${ }^{a}$ & Function $^{\mathrm{b}}$ \\
\hline Aldo/keto reductase family protein & AT2G37770 & BQ119151 & Carbohydrate metabolism \\
\hline Putative pectin methylesterase & AT1G11580 & BQ508460 & Cell wall \\
\hline Putative aminotransferase & AT2G24850 & BQ518210 & Metal handling \\
\hline Putative cytochrome P450 & AT2G45570 & BQ510046 & Miscellaneous \\
\hline Putative phospholipase & AT2G39420 & BQ516784 & Not assigned \\
\hline Glucose- 6- phosphate 1- dehydrogenase & AT1G24280 & BQ508597 & Oxidative phosphorylation \\
\hline Similar to zinc metalloproteinases & AT1G24140 & BQ111463 & Protein.degradation \\
\hline Ubiquitin activating enzyme- like protein & AT4G24940 & BQ112099 & Protein.degradation \\
\hline Polyubiquitin protein & At5g20620 & BQ514904 & Protein.degradation \\
\hline Ubiquitin family protein & & BQ518413 & Protein.degradation \\
\hline Hsc70 protein & AT5G02490 & BQ505871 & Protein.folding \\
\hline Putative Ser/ The phophatase 2C & AT4G08260 & BQ112763 & Protein.modification \\
\hline Putative copper/ zinc superoxide dismutase & AT1G12520 & BQ114448 & Redox \\
\hline Thioredoxin $\mathrm{h}$ & AT1G45145 & BQ510896 & Redox \\
\hline Putative glutathione $S$ - transferase & AT2G02930 & BQ515272 & Redox \\
\hline Putative glutathione S- transferase & AT4G02520 & BQ515272 & Redox \\
\hline Protein disulfide- isomerase- like protein & AT3G54960 & BQ116026 & Redox \\
\hline WRKYG & AT1G62300 & BQ517451 & RNA.transcription \\
\hline SRG1 & AT1G17020 & BQ517001 & Secondary metabolism \\
\hline Putative ligand- gated ion channel protein & AT2G29120 & BQ510910 & Signaling \\
\hline Putative receptor- like protein kinase & AT2G31880 & BQ505516 & Signaling \\
\hline Putative calcium binding protein & AT2G43290 & BQ518008 & Signaling calcium \\
\hline Calreticulin & AT1G08450 & BQ111561 & Signaling.calcium \\
\hline Calmodulin & AT2G41110 & BQ511870 & Signaling.calcium \\
\hline Heat shock protein 70 & AT3G12580 & BQ112515 & Stress.abiotic \\
\hline Late embryogenesis abundant protein homolog & AT4G02380 & BQ113428 & Stress.abiotic \\
\hline Heat shock protein 83 & AT5G52640 & BQ511515 & Stress.abiotic \\
\hline Thaumatin- like protein & AT1G75040 & BQ515360 & Stress.biotic \\
\hline Pathogenesis- related PR- 1- like protein & AT2G14610 & BQ121920 & Stress.biotic \\
\hline Putative endochitinase & AT2G43570 & BQ118729 & Stress.biotic \\
\hline Class IV chitinase & AT3G54420 & BQ516435 & Stress.biotic \\
\hline Beta- 1,3-glucanase & AT3G57260 & BQ511760 & Stress.biotic \\
\hline
\end{tabular}

${ }^{a}$ S. tuberosum designation is given, because $N$. benthamiana data were collected using the potato microarray from TIGR (Rensink and Buell 2005). The genes highlighted in bold share significant sequence similarity in BLAST searches $\left(E<e^{-20}\right)$ but are not necessarily orthologous. The other genes share a common description between Arabidopsis and potato, but sequence similarity is low $\left(\mathrm{E}>\mathrm{e}^{-10}\right)$.

${ }^{\mathrm{b}}$ Function based on Arabidopsis annotation using the MAPMAN program (Thimm et al. 2004). 
pression of defense-related genes when expressed from CMV or as a transgene. In Nicotiana species, it is apparent that the induction of SA-dependent defenses is detrimental to compatible interactions with CMV or TMV. Thus, Cmv2b may promote CMV accumulation in Nicotiana species by antagonizing SAdependent defenses.

In light of the potentially antagonistic effects of $\mathrm{Cmv} 2 \mathrm{~b}$ to $\mathrm{SA}$, it may be significant that CMV causes changes in defenserelated gene expression of much less magnitude than ORMV (a tobamovirus) in Arabidopsis plants despite the rapid and abundant accumulation of each virus (Huang et al. 2005; Whitham et al. 2003). Perhaps this difference is due to the activity of Cmv2b. Deducing the role of Cmv2b in suppressing SAmediated defenses is complicated by the fact that it is also an RNA silencing suppressor (RSS) (Brigneti et al. 1998). It is possible that it is a multifunctional protein that is both an antagonist of SA signaling and an RSS or, alternatively, one of the activities may mediate the other. The ability of $\mathrm{Cmv} 2 \mathrm{~b}$ to interfere with SA-mediated defenses is apparently not a general property of RSS, because potyvirus HC-Pro, a potent silencing suppressor, causes enhanced resistance to some pathogens, including viruses and oomycetes (Pruss et al. 2004). The potential that other viruses also antagonize SA-dependent defenses is relatively unexplored, and such lines of investigation could provide additional insight into counter-defensive strategies utilized by plant viruses.

CaMV induces some other potentially interesting changes in host gene expression associated with plant defenses in addition to SA-mediated expression of $P R$ genes. Infection of Arabidopsis with CaMV virions but not its infectious DNA results in rapid and systemic induction of both PDF1.2 and GST1 (Love et al. 2005). In contrast, $P D F 1.2$ is not induced in compatible interactions with RNA viruses, such as ORMV, CMV, or Turnip mosaic virus (TuMV), and although GST1 (as well as other GST) is induced by these viruses, it is not induced until later in infection (Huang et al. 2005; Whitham et al. 2003). Expression of GST1 in response to CaMV was reduced or inhibited in etrl-1 and rbohD/rbohF mutants, indicating a dependence on ethylene signaling and NADPH oxidase for its induction. In addition, reactive oxygen species were induced systemically, and they were dependent on ethylene and NADPH oxidase for their accumulation. Despite the fact that etrl-1 or $r b o h D / r b o h F$ mutants do not develop systemic induction of reactive oxygen species and GSTI expression is inhibited, there is no enhanced susceptibility to CaMV infection. This result implies that the response is trivial or that CaMV might have the ability to suppress the defense response. In either scenario, loss of this defense pathway would not lead to enhanced susceptibility. Love and associates (2005) posited that the role of CaMV virions in inducing this defenselike response has some similarity to the activity of pathogen-associated molecular patterns (PAMP) in Arabidopsis. A PAMP, in general, is a molecular signature that is unique to a type of pathogen and activates receptor-mediated defenses in a host organism. In the case of CaMV, the PAMP is predicted to be some peptide located in the capsid of the virion. Interestingly, TMV coat protein also induces a rapid induction of reactive oxygen species in epidermal cells of compatible tobacco (Allan et al. 2001), which suggests PAMPlike activity for it as well. It will be interesting to determine what other viruses possess PAMP-like activity and if these activities influence the outcome of virus-host interactions. Certainly, if these defense responses are capable of limiting viral infection, it would suggest that viruses need to carry some sort of counter-defensive function in their arsenal.

To date, only two studies have been published on the transcriptome of an incompatible virus-host interaction (Ishihara et al. 2004; Marathe et al. 2004). The target of both studies was the $R C Y 1$ gene of Arabidopsis (ecotype C24) that confers a hypersensitive resistance response to CMV-Y (Takahashi et al. 2001). RCYI encodes a member of the coiled coil-nucleotide binding site-leucine rich repeat class of resistance gene proteins and is dependent on SA, jasmonic acid (JA), and ethylene (ET) for an effective resistance response (Takahashi et al. 2002). An infection timecourse covering the first $0,3,5$, and $18 \mathrm{~h}$ after CMV-Y infection of resistant $R C Y 1 \mathrm{C} 24$ plants was analyzed using the Arabidopsis ATH1 genome array (Marathe et al. 2004). Statistical analysis of the data identified 444 genes that responded to CMV-Y infection at the estimated false discovery rate of 5\%. Ishihara and associates (2004) profiled gene expression in the resistant $\mathrm{C} 24$ and susceptible Col-0 ecotypes at $6,12,24$, and $48 \mathrm{~h}$ after infection, using a macroarray representing approximately 9,000 EST. They observed that similar numbers of genes (50 to 80 ) were modulated significantly in the two ecotypes through the first $24 \mathrm{~h}$ but, by $48 \mathrm{~h}$, the response to $\mathrm{CMV}-\mathrm{Y}$ was much more robust in resistant $\mathrm{C} 24$ plants (788 significant genes) versus susceptible Col-0 plants (150 significant genes). The results of Marathe and associates (2004) suggest that the resistance response to CMV-Y is more robust at the earlier timepoints, but these apparent differences may be due to the numbers of genes on the arrays and the sensitivity of the gene expression assays using different technologies. Surprisingly, only a few of the significant genes were identified in common between these two studies. The common genes included five of unknown function (At1g19180, At1g70700, At3g07090, At4g01870, At5g48180), a calcium binding protein (At2g33380), a Cf2-like gene (At2g32680), a serine/threonine kinase (At4g23140), a superoxide dismutase (At4g25100), and a plasma membrane ATPase (At4g37640). The overall lack of similarity between the two studies is probably due to several complicating factors. The Ishihara study (Ishihara et al. 2004) used an array covering the partial genome and excluded many genes in order to correct for comparing gene expression between the Col-0 and C24 ecotypes. In addition, the Marathe study (Marathe et al. 2004) used a shorter timecourse prior to the onset of a more robust response observed later by Ishihara and associates (2004).

Induction of HSP. HSP perform diverse tasks in response to cellular stress as well as in normal growth and development, and diverse DNA and RNA viruses induce their expression almost ubiquitously in eukaryotic hosts (Allen and Nuss 2004; Allen et al. 2003; Aranda et al. 1996; Colberg-Poley and Santomenna 1988; Escaler et al. 2000b; Huber 1992; Kao and Nevins 1983; Phillips et al. 1991; Sedger and Ruby 1994; Senthil et al. 2005; Whitham et al. 2003). The induction of HSP70 in plant cells in response to a viral pathogen was first demonstrated by in situ hybridization in pea embryos (Aranda et al. 1996; Escaler et al. 2000b). Since then, microarray analyses of interactions between five different positive stranded RNA viruses and Arabidopsis leaves provided further evidence that the induction of heat shock genes in response to viral infection is indeed a common occurrence (Whitham et al. 2003). In addition, this study extended the list of virus-induced HSP to members of the small HSP, HSP70, HSP90, and HSP100 families of HSP. Interestingly, several of these genes, including HSP17.4, HSP17.6, HSP23.6, HSP70, HSP83, and HSP101 were induced very early in infection by the tobamoviruses ORMV and TVCV, as compared with that by CMV, Potato virus $X$, or TuMV. As shown in Table 2, HSP70 and HSP83 are also induced in N. benthamiana in response to the negative-strand RNA viruses, SYNV, and INSV. INSV, which is a more aggressive virus in $N$. benthamiana, also induces the expression of small HSP and other HSP70 homologs (Senthil et al. 2005).

In both plants and animals, the accumulation of HSP mRNA is controlled largely at the level of transcription in response to 
stress such as heat shock and in growth and development (Schoffl et al. 1998). Following heat stress, heat shock transcription factors oligomerize and bind to well-defined promoter sequences termed heat shock elements (HSE). The consensus HSE in plants is 5'-GAANNTTCNNGAA-3' (Barros et al. 1992), which is similar to consensus eukaryotic HSE comprised of the core sequence 5'-NGAAN-3' (Schoffl et al. 1998). The current evidence in plants and animals indicates that HSE alone are not sufficient for the expression of heat shock genes in response to viral infection (Aranda et al. 1999; Phillips et al. 1991; Whitham et al. 2003). Furthermore, the expression of HSP101 and HSP70 was found to be independent of the SA, JA, and ET defense signaling pathways, demonstrating that their transcript accumulation is not coregulated with defenserelated genes (Carr et al. 2006). These studies indicate that viruses activate the expression of HSP by an uncharacterized mechanism in both plants and animals.

The induction of HSP might occur by either general or specific mechanisms. The adenoviral protein Gam1 was the first viral protein demonstrated to be a specific elicitor of the expression of HSP70, HSP27, and HSP40, but not HSP90 $\alpha$ or HSC70 (Glotzer et al. 2000). In plants, a specific viral elicitor of HSP70 expression has not been identified; however, the capsid protein and two vector transmission proteins encoded by RNA 2 of Pea early browning virus (PEBV) were ruled out (Escaler et al. 2000a). RNA 1 of PEBV alone was sufficient for induction of HSP70 expression in pea cotyledons. Thus, any of the replicase, movement, or $16-\mathrm{kDa}$ proteins encoded by RNA1 could be the elicitor. This conclusion should be considered with caution, due to recent evidence that certain $N$. benthamiana genes encoding primarily cytoplasmic HSP can be induced by high levels of expression of individual viral proteins (Aparicio et al. 2005). A response to the accumulation of unfolded proteins in the cytoplasm was proposed to occur as high levels of heterologous proteins, such as those of viral origin, accumulate. Thus, in the case of $\mathrm{PEBV}$, the levels of replicase, movement, and 16-kDa proteins, alone or in combination, in infected cells might be sufficient to induce a general response in the absence of the RNA2-encoded proteins. Jockusch and associates (2001) also implicated accumulation of aggregated viral proteins as potent inducers of HSP expression. They found that TMV strains with mutant capsid proteins, which denatured at slightly elevated temperatures, induced tobacco HSP expression to levels above infection by a wild-type TMV strain. In support of this role for the tobamovirus capsid protein, we have recently found that ORMV capsid protein alone is sufficient to induce expression of HSP70 and HSP101 mRNA (Carr et al. 2006).

The importance of HSP70 and other HSP as host factors that facilitate viral infection is becoming increasingly clear (Glotzer et al. 2000; Nagy and Pogany 2006; Serva and Nagy 2006; Sullivan and Pipas 2001). For example, HSP70 family members in yeast are associated with Tomato bushy stunt virus replicase complexes and deletion or overexpression of these HSP70 family members results in inhibition or enhancement, respectively, of viral replication (Serva and Nagy 2006). Further support for the significance of HSP in viral infection is that plant and animal viruses encode HSP homologs in their genomes or possess proteins that recruit host HSP. The plant pathogenic closteroviruses are positive-stranded RNA viruses that encode an HSP70 homolog, HSP70h (Agranovsky et al. 1998), which has been shown to function in virion assembly (Satyanarayana et al. 2000) and cell-to-cell movement (Alzhanova et al. 2000; Peremyslov et al. 1999). Although no animal virus has been shown to encode an HSP70 homolog, host HSP70 can be recruited through interactions with virusencoded J-domain proteins (Kelley 1998).
Common responses of plants to viruses:

\section{Developmental defects.}

Many of the studies highlighted in Table 1 identified genes for which there is no obvious connection to plant stress or defense responses. Some of these genes may have direct connections to the developmental defects (i.e., the disease symptoms) that often result from viral infection. Misregulation of genes involved in plant growth and development is expected to lead to symptoms (Fig. 1). The ability of viruses to perturb or interfere in signaling pathways involving phytohormones and regulatory small RNAs provides opportunities for them to interfere with host gene expression and affect plant growth and development.

Viral infection and phytohormone biosynthesis and signaling. The abnormal growth forms of virus-infected plants have encouraged experiments that examine the effects of viruses on hormone levels in plants and vice versa (Jameson 2000). While it is difficult to formulate general rules about the effects of viruses on phytohormones, it is clear that abscisic acid, auxin, cytokinin, giberellin, and ethylene levels, alone or in combination, can all be perturbed, depending on the virus-host combination. Recently, direct links between auxin signaling and giberellin levels have been established for TMV and Rice dwarf virus (RDV), respectively.

The helicase domain of the TMV 126- and 183-kDa replicase proteins was shown to interact with the Aux/indole 3-acetic acid (IAA) transcription factor IAA26 (Padmanabhan et al. 2005). A single amino-acid mutation (V1087I) in the helicase domain abolished the interaction between the replicase proteins and IAA26. Even though this interaction was disrupted, the TMV mutant replicated and moved systemically in Arabidopsis and tobacco plants at levels equivalent to the wild-type virus. Interestingly, the symptoms of infection were attenuated indicating that TMV replication and movement could be partially uncoupled from symptoms in both Arabidopsis and $N$. benthamiana. Thus, this interaction, while contributing to the symptoms, is apparently not required for a successful TMV infection in Arabidopsis or $N$. benthamiana. Silencing of IAA26 resulted in plants with phenotypes similar to those infected with TMV, indicating that TMV replicase also causes a loss of IAA26 function. This was verified, in part, by showing that TMV infection redirects IAA26 from the nucleus to the cytoplasm.

The ability of TMV to modulate symptoms through interaction with IAA26 was postulated to be due to interference with its normal role in forming heterodimers with auxin-response factors (ARF) (Padmanabhan et al. 2005). In the absence of auxin, Aux/IAA proteins like IAA26 bind to ARF transcription factors and prevent them from modulating the transcription of auxin-responsive genes. However, in the presence of auxin, the Aux/IAA proteins are targeted for degradation, thus freeing the ARF proteins to modulate transcription of their target genes. The TMV replicase thus functions as an auxin mimic by diverting IAA26 from the nucleus and allowing its target ARF to modulate transcription of their target genes. In support of this idea, the authors observed that $30 \%$ (20 of 66) of genes that were significantly altered in their expression in response to TMV in Arabidopsis (Golem and Culver 2003) also possessed two or more auxin-response elements (ARE; TGTCTC) in their promoters. Genes possessing this motif in their promoters are part of the early response to auxin and are subject to transcriptional control by Aux/IAA and ARF proteins. Five of the 20 genes that respond to TMV and possess at least two ARE were tested for their response to auxin and were shown to exhibit similar patterns of expression in response to auxin and TMV. Because IAA26 is one of 29 members of a gene family, it will be interesting to see how TMV interacts with other 
members of this family and if any of these interactions have additional effects on symptoms, host gene expression, or viral infection itself.

In addition to affecting auxin signaling events, viral proteins have also been identified that affect giberellic acid (GA) signaling. One such example is the $\mathrm{P} 2$ protein of RDV, which currently has no known role in the infection process except that it is a symptom determinant. Recently, Zhu and associates (2005) demonstrated that P2 interacts with four different entkaurene oxidases or oxidaselike proteins by yeast two-hybrid assay. ent-kaurene oxidases catalyze a step in the synthesis of GA, and ent-kaurene oxidase mutant rice plants are deficient in GA. In addition, this mutant rice possesses a phenotype similar to symptoms caused by RDV infection. The authors demonstrated that RDV infection results in decreased GA levels and symptoms that could be alleviated by GA application. Although host gene expression assays were not performed, it is likely that GA responsive genes are modulated by RDV infection. Despite the role of RDV P2 in disrupting GA levels in rice, the authors were unable to conclude that this interaction has a significant effect on infection, suggesting that it may be coincidental but detrimental to the plant.

Viral RSS. The activities of viral RSS have recently received a great deal of attention and have been the target of numerous excellent reviews that have included descriptions of their effects on host gene expression (Dunoyer and Voinnet 2005). These proteins promote infections by interfering with an intrinsic host defense mechanism and simultaneously interfere with regulatory small RNAs, such as micro RNAs (miRNAs) and trans-acting small interfering RNAs, which lead to defects in host growth and development. Because the targets of miRNAs are frequently transcription factors, the RSS can lead to misexpression of these genes and their targets. Currently, there is not much information available about the target genes that are transcriptionally controlled by these small RNA-regulated transcription factors, but we expect that such genes are present in the microarray data sets from virus-infected plants.

Most studies of RSS function have centered on the activities of suppressors encoded by plus-strand RNA viruses as opposed to those present in negative or ambisense RNA genomes. Given that these latter viruses, namely, tospoviruses, tenuiviruses, and rhabdoviruses, replicate in their insect vectors, the identification of their cognate RSS proteins is of particular interest, since they must evade antiviral responses in plant and animal cells. The first report of an RSS encoded by a negative-sense/ ambisense RNA was the NSs protein of Tomato spotted wilt virus (TSWV, a tospovirus) (Takeda et al. 2002). Using a green fluorescent protein (GFP)-based transient suppression assay, it was determined that NSs could suppress sense transgeneinduced posttranscriptional gene silencing (PTGS) but not inverted repeat transgene-induced PTGS (Takeda et al. 2002). Later studies showed that the NSs and NS3 proteins of TSWV and Rice hoya blanca virus (a tenuivirus), which are encoded in genetically analogous positions, are RSS proteins (Bucher et al. 2003). Evidence that plant rhabdoviruses also encode RSS proteins is just starting to emerge (M. M. Goodin, unpublished data). Characterization of these proteins is of particular interest, because the Nucleorhabdovirus and Cytorhabdovirus genera have members that replicate in nuclei or cytoplasmic loci, respectively. This is in contrast to plant plus-strand RNA viruses and negative-strand tospoviruses and tenuiviruses, which all replicate in the cytoplasm. Therefore, a comparison of the mode of action of rhabdoviral RSS proteins may yield significant insight into the adaptations that have occurred in genetically related viruses during their evolution and differentiation into nucleoplasmic and cytoplasmic species.

\section{Future prospects.}

Global proteome profiling and targeted proteomics approaches can provide information that is complementary to transcriptional profiling by providing a more accurate view of the effects of viral infection on the host proteome (Korth et al. 2005). Global protein profiling has not yet been applied extensively to plant virus interactions. Two-dimensional gel electrophoresis was used to separate proteins from rice cellular suspensions that were infected with Rice yellow mottle virus (RYMV) (Ventelon-Debout et al. 2004). This analysis identified proteins with altered accumulation in response to RYMV in susceptible and partially resistant rice cultivars. Protein spots that were significantly altered in response to RYMV in the two cultivars were digested with trypsin and were analyzed by mass spectrometry for identification. The three major classes of proteins identified were defense- and stress-related proteins, translation and protein turnover, and metabolic proteins. These results indicate that, with respect to cellular stress, similar events are happening at the protein and mRNA levels, but much more work in this area is needed to more thoroughly establish positive or negative correlations between mRNA and protein levels. In addition to altered expression of mRNA transcripts or protein levels, another important factor to consider will be relocalization of host proteins through protein-protein interactions with viral proteins. The examples provided by TMV and Aux/IAA and RDV and ent-kaurene oxidase illustrate the impact that viral proteins have when they sequester host proteins and prevent them from performing their normal functions. Differential localization of host proteins in response to virus infection will probably not be restricted to genes with altered expression, so many will need to be identified through targeted proteomics approaches utilizing viral proteins as bait.

Currently, we do not know the impact that host gene-expression changes have on viral pathogenesis. One intriguing question that arises is in regard to the induction of basal defense responses. It is well-established that RSS play important roles in counteracting the RNA silencing mechanism that would normally function to limit viral infections, but are other induced defenses also important factors for viruses to deal with? It is possible that there are layers of defense that viruses must overcome, including basal SA-dependent defenses and RNA silencing. Perhaps viruses are successful in suppressing both types of defense. By some unknown mechanism, compatible viruses induce SA-dependent defenselike responses in Arabidopsis. How these responses are triggered as a general consequence of infection is an interesting question. Somewhat puzzling is that Arabidopsis mutants defective in SA-mediated defense responses have not been found to have enhanced susceptibility to wildtype viruses. In contrast, a tomato eds 1 (enhanced disease susceptibility 1) mutant was reported to be more susceptible to TMV, suggesting that there can be a role for basal-induced defenses (Hu et al. 2005). If basal-induced defenses do indeed have the capacity to limit viral infection, then it would seem logical that viruses might suppress them.

There is still much information lacking regarding the effects of each protein in triggering host responses and the signaling networks that are involved. In addition, for most viral proteins, the identities of the different host proteins that are targets for interaction and that participate in viral pathogenesis remain unknown. Host genes with altered expression profiles provide candidates for reverse genetic experiments to test for roles in viral pathogenesis and provide insight into the signaling pathways that are modulated by viral infection. Targeted proteomic approaches along with mRNA and proteome profiling will continue to provide new insights into host-virus interactions. When combined with engineered viruses or studies on individual viral proteins, it will be possible to understand the cause 
and effect relationships of each viral protein on host physiology. A growing number of tools and resources make these reverse genetic studies possible in a variety of hosts in addition to Arabidopsis. Most of the genomic studies between hosts and viruses have been performed using dicot hosts. As demonstrated in Table 2, it is clear that there are conserved responses to positive- and negative-stranded RNA viruses among dicots, and it will be interesting to see if this extends to monocot hosts. It will also be important to do large-scale meta analyses that compare host responses to viruses and other pathogens, such as bacteria, fungi, and viroids. These analyses will reveal unique and conserved effects of viruses on their hosts in comparison to other microbial pathogens, as they engage signaling pathways leading to disease and defense.

\section{ACKNOWLEDGMENTS}

This work was supported by the United States Department of Agriculture (USDA)-National Research Initiative (02-35319-12566) and Research Grant Award Number US-3623-04 from BARD, the United States-Israel Binational Agricultural Research and Development Fund awarded to S. A. Whitham, USDA/ Cooperative State Research Education and Extension Service (2005-01612) awarded to M. Goodin, and Hatch Act and State of Iowa Funds. This manuscript is published with the approval of the Director of the Kentucky Agricultural Experiment Station as Journal Article (06-12096).

\section{LITERATURE CITED}

Agranovsky, A. A., Folimonov, A. S., Folimonova, S., Morozov, S., Schiemann, J., Lesemann, D., and Atabekov, J. G. 1998. Beet yellows closterovirus HSP70-like protein mediates the cell-to-cell movement of a potexvirus transport-deficient mutant and a hordeivirus-based chimeric virus. J. Gen. Virol. 79:889-895.

Allan, A. C., Lapidot, M., Culver, J. N., and Fluhr, R. 2001. An early tobacco mosaic virus-induced oxidative burst in tobacco indicates extracellular perception of the virus coat protein. Plant Physiol. 126:97-108.

Allen, T. D., and Nuss, D. L. 2004. Specific and common alterations in host gene transcript accumulation following infection of the chestnut blight fungus by mild and severe hypoviruses. J. Virol. 78:4145-4155.

Allen, T. D., Dawe, A. L., and Nuss, D. L. 2003. Use of cDNA microarrays to monitor transcriptional responses of the chestnut blight fungus Cryphonectria parasitica to infection by virulence-attenuating hypoviruses. Eukaryot. Cell 2:1253-1265.

Alzhanova, D. V., Hagiwara, Y., Peremyslov, V. V., and Dolja, V. V. 2000. Genetic analysis of the cell-to-cell movement of Beet yellows closterovirus. Virology 268:192-200.

Aparicio, F., Thomas, C. L., Lederer, C., Niu, Y., Wang, D., and Maule, A. J. 2005. Virus induction of heat shock protein 70 reflects a general response to protein accumulation in the plant cytosol. Plant Physiol. 138:529-536.

Aranda, M. A., Escaler, M., Wang, D., and Maule, A. J. 1996. Induction of HSP70 and polyubiquitin expression associated with plant virus replication. Proc. Natl. Acad. Sci. U.S.A. 93:15289-15293.

Aranda, M. A., Escaler, M., Thomas, C. L., and Maule, A. J. 1999. A heat shock transcription factor in pea is differentially controlled by heat and virus replication. Plant J. 20:153-161.

Barros, M. D., Czarnecka, E., and Gurley, W. B. 1992. Mutational analysis of a plant heat shock element. Plant Mol. Biol. 19:665-675.

Brigneti, G., Vionnet, O., Li, W.-X., Ji, L.-H., Ding, S.-W., and Baulcombe, D. C. 1998. Viral pathogenicity determinants are suppessors of transgene silencing in Nicotiana benthamiana. EMBO (Eur. Mol. Biol. Organ.) J. 17:6739-6746.

Bucher, E., Sijen, T., De Haan, P., Goldbach, R., and Prins, M. 2003. Negative-strand tospoviruses and tenuiviruses carry a gene for a suppressor of gene silencing at analogous genomic positions. J. Virol. 77:1329-1336.

Carr, T., Wang, Y., Huang, Z., Yeakley, J. M., Fan, J. B., and Whitham, S. A. 2006. Tobamovirus infection is independent of HSP101 mRNA induction and protein expression. Virus Res. 121:33-41.

Chen, W., Provart, N., Glazebrook, J., Katagiri, F., Chang, H. S., Eulgem, T., Mauch, F., Luan, S., Zou, G., Whitham, S. A., Budworth, P. R., Tao, Y., Xie, Z., Chen, X., Lam, S., Kreps, J. A., Harper, J., Si-Ammour, A., Mauch-Mani, B., Heinlein, M., Kobayashi, K., Hohn, T., Dangl, J. L., Wang, X., and Zhu, T. 2002. Expression profile matrix of Arabidopsis transcription factor genes implies their putative functions in response to environmental stresses. Plant Cell 14:559-574.

Colberg-Poley, A. M., and Santomenna, L. D. 1988. Selective induction of chromosomal gene expression by human cytomegalovirus. Virology $166: 217-228$.

Cooper, B. 2001. Collateral gene expression changes induced by distinct plant viruses during the hypersensitive resistance reaction in Chenopodium amaranticolor. Plant J. 26:339-349.

Dempsey, D. A., Pathirana, M. S., Wobbe, K. K., and Klessig, D. F. 1997. Identification of an Arabidopsis locus required for resistance to Turnip crinkle virus. Plant J. 11:301-311.

Dunoyer, P., and Voinnet, O. 2005. The complex interplay between plant viruses and host RNA-silencing pathways. Curr. Opin. Plant Biol. 8:415-423.

Escaler, M., Aranda, M. A., Roberts, I. M., Thomas, C. L., and Maule, A J. 2000a. A comparison between virus replication and abiotic stress (heat) as modifiers of host gene expression in pea. Mol. Plant Pathol. 1:159-167.

Escaler, M., Aranda, M. A., Thomas, C. L., and Maule, A. J. 2000b. Pea embryonic tissues show common responses to the replication of a wide range of viruses. Virology 267:318-325.

Fregene, M., Matsumura, H., Akano, A., Dixon, A., and Terauchi, R. 2004. Serial analysis of gene expression (SAGE) of host-plant resistance to the cassava mosaic disease (CMD). Plant Mol. Biol. 56:563-571.

Gaffney, T., Friedrich, L., Vernooij, B., Negrotto, D., Nye, G., Uknes, S., Ward, E., Kessmann, H., and Ryals, J. 1993. Requirement of salicylic acid for the induction of systemic acquired resistance. Science 261:754756.

Geri, C., Cecchini, E., Giannakou, M. E., Covey, S. N., and Milner, J. J. 1999. Altered patterns of gene expression in Arabidopsis elicited by Cauliflower mosaic virus (CaMV) infection and by a CaMV gene VI transgene. Mol. Plant Microbe Interact. 12:377-384.

Glotzer, J. B., Saltik, M., Chiocca, S., Michou, A. I., Moseley, P., and Cotten, M. 2000. Activation of heat-shock response by an adenovirus is essential for virus replication. Nature 407:207-211.

Golem, S., and Culver, J. N. 2003. Tobacco mosaic virus induced alterations in the gene expression profile of Arabidopsis thaliana. Mol. Plant Microbe Interact. 16:681-688.

Havelda, Z., and Maule, A. J. 2000. Complex spatial responses to Cucumber mosaic virus infection in susceptible Cucurbita pepo cotyledons. Plant Cell 12:1975-1986.

Hu, G., deHart, A. K., Li, Y., Ustach, C., Handley, V., Navarre, R., Hwang, C. F., Aegerter, B. J., Williamson, V. M. and Baker, B. 2005. EDS1 in tomato is required for resistance mediated by TIR-class $R$ genes and the receptor-like $R$ gene Ve. Plant J. 42:376-391.

Huang, Z., Yeakley, J. M., Garcia, E. W., Holdridge, J. D., Fan, J. B., and Whitham, S. A. 2005. Salicylic acid-dependent expression of host genes in compatible Arabidopsis-virus interactions. Plant Physiol. 137:11471159 .

Huber, S. A. 1992. Heat-shock protein induction in adriamycin and picornavirus-infected cardiocytes. Lab. Invest. 67:218-224.

Ishihara, T., Sakurai, N., Sekine, K. T., Hase, S., Ikegami, M., Shibata, D., and Takahashi, H. 2004. Comparative analysis of expressed sequence tags in resistant and susceptible ecotypes of Arabidopsis thaliana infected with Cucumber mosaic virus. Plant Cell Physiol. 45:470-480.

Itaya, A., Matsuda, Y., Gonzales, R. A., Nelson, R. S., and Ding, B. 2002. Potato spindle tuber viroid strains of different pathogenicity induces and suppresses expression of common and unique genes in infected tomato. Mol. Plant Microbe Interact. 15:990-999.

Jameson, P. 2000. Cytokinins and auxins in plant-pathogen interactionsAn overview. Plant Growth Regul. 32:369-380.

Jenner, R. G., and Young, R. A. 2005. Insights into host responses against pathogens from transcriptional profiling. Nat. Rev. Microbiol. 3:281294.

Ji, L. H., and Ding, S. W. 2001. The suppressor of transgene RNA silencing encoded by Cucumber mosaic virus interferes with salicylic acidmediated virus resistance. Mol. Plant Microbe Interact. 14:715-724.

Jockusch, H., Wiegand, C., Mersch, B., and Rajes, D. 2001. Mutants of Tobacco mosaic virus with temperature-sensitive coat proteins induce heat shock response in tobacco leaves. Mol. Plant Microbe Interact. 14:914-917.

Kachroo, P., Yoshioka, K., Shah, J., Dooner, H. K., and Klessig, D. F. 2000. Resistance to Turnip crinkle virus in Arabidopsis is regulated by two host genes and is salicylic acid dependent but NPR1, ethylene, and jasmonate independent. Plant Cell 12:677-690.

Kao, H. T., and Nevins, J. R. 1983. Transcriptional activation and subsequent control of the human heat shock gene during adenovirus infection. Mol. Cell Biol. 3:2058-2065.

Kelley, W. L. 1998. The J-domain family and the recruitment of chaperone power. Trends Biochem. Sci. 23:222-227.

Korth, M. J., Kash, J. C., Furlong, J. C., and Katze, M. G. 2005. Virus in- 
fection and the interferon response: A global view through functional genomics. Methods Mol. Med. 116:37-55.

Laird, J., Armengaud, P., Giuntini, P., Laval, V., and Milner, J. J. 2004. Inappropriate annotation of a key defence marker in Arabidopsis: Will the real PR-1 please stand up? Planta 219:1089-1092.

Love, A. J., Yun, B. W., Laval, V., Loake, G. J., and Milner, J. J. 2005. Cauliflower mosaic virus, a compatible pathogen of Arabidopsis, engages three distinct defense-signaling pathways and activates rapid systemic generation of reactive oxygen species. Plant Physiol. 139:935948 .

Malamy, J., Carr, J. P., Klessig, D. F., and Raskin, I. 1990. Salicylic acid: A likely endogenous signal in the resistance response of tobacco to viral infection. Science 250:1002-1004.

Malamy, J., Hennig, J., and Klessig, D. F. 1992. Temperature-dependent induction of salicylic acid and its conjugates during the resistance response to Tobacco mosaic virus infection. The Plant Cell 4:359-366.

Marathe, R., Guan, Z., Anandalakshmi, R., Zhao, H., and Dinesh-Kumar, S. P. 2004. Study of Arabidopsis thaliana resistome in response to cucumber mosaic virus infection using whole genome microarray. Plant Mol. Biol. 55:501-520.

Maule, A., Leh, V., and Lederer, C. 2002. The dialogue between viruses and hosts in compatible interactions. Curr. Opin. Plant Biol. 5:279-284.

Nagy, P. D., and Pogany, J. 2006. Yeast as a model host to dissect functions of viral and host factors in tombusvirus replication. Virology 344:211-220.

Padmanabhan, M. S., Goregaoker, S. P., Golem, S., Shiferaw, H., and Culver, J. N. 2005. Interaction of the tobacco mosaic virus replicase protein with the Aux/IAA protein PAP1/IAA26 is associated with disease development. J. Virol. 79:2549-2558.

Peremyslov, V. V., Hagiwara, Y., and Dolja, V. V. 1999. HSP70 homolog functions in cell-to-cell movement of a plant virus. Proc. Natl. Acad. Sci. U.S.A. 96:14771-14776.

Phillips, B., Abravaya, K., and Morimoto, R. I. 1991. Analysis of the specificity and mechanism of transcriptional activation of the human hsp70 gene during infection by DNA viruses. J. Virol. 65:5680-5692

Pruss, G. J., Lawrence, C. B., Bass, T., Li, Q. Q., Bowman, L. H., and Vance, V. 2004. The potyviral suppressor of RNA silencing confers enhanced resistance to multiple pathogens. Virology 320:107-120.

Rensink, W. A. and Buell, C. R. 2005. Microarray expression profiling resources for plant genomics. Trends Plant Sci. 10:603-609.

Rensink, W. A., Lee, Y., Liu, J., Iobst, S., Ouyang, S. and Buell, C. R. 2005. Comparative analyses of six solanaceous transcriptomes reveal a high degree of sequence conservation and species-specific transcripts. BMC Genomics 6:124.

Robatzek, S., and Somssich, I. E. 2001. A new member of the Arabidopsis WRKY transcription factor family, AtWRKY6, is associated with both senescence- and defence-related processes. Plant J. 28:123-133.

Robatzek, S., and Somssich, I. E. 2002. Targets of AtWRKY6 regulation during plant senescence and pathogen defense. Genes Dev. 16:11391149.

Ryals, J. A., Neuenschwander, U. H., Willits, M. G., Molina, A., Steiner, H.-Y., and Hunt, M. D. 1996. Systemic acquired resistance. Plant Cell 8:1809-1819.

Satyanarayana, T., Gowda, S., Mawassi, M., Albiach-Marti, M. R., Ayllon, M. A., Robertson, C., Garnsey, S. M., and Dawson, W. O. 2000. Clos- terovirus encoded HSP70 homolog and p61 in addition to both coat proteins function in efficient virion assembly. Virology 278:253-265.

Schoffl, F., Prandl, R., and Reindl, A. 1998. Regulation of the heat-shock response. Plant Physiol 117:1135-1141.

Sedger, L., and Ruby, J. 1994. Heat shock response to vaccinia virus infection. J. Virol. 68:4685-4689.

Senthil, G., Liu, H., Puram, V. G., Clark, A., Stromberg, A., and Goodin, M. M. 2005. Specific and common changes in Nicotiana benthamiana gene expression in response to infection by enveloped viruses. J. Gen. Virol. 86:2615-2625.

Serva, S., and Nagy, P. D. 2006. Proteomics analysis of the tombusvirus replicase: Hsp70 molecular chaperone is associated with the replicase and enhances viral RNA replication. J. Virol. 80:2162-2169.

Sullivan, C. S., and Pipas, J. M. 2001. The virus-chaperone connection. Virology 287:1-8.

Takahashi, H., Suzuki, M., Natsuaki, K., Shigyo, T., Hino, K., Teraoka, T. Hosokawa, D., and Ehara, Y. 2001. Mapping the virus and host genes involved in the resistance response in Cucumber mosaic virus-infected Arabidopsis thaliana. Plant Cell Physiol. 42:340-347.

Takahashi, H., Miller, J., Nozaki, Y., Takeda, M., Shah, J., Hase, S. Ikegami, M., Ehara, Y., and Dinesh-Kumar, S. P. 2002. RCY1, an Arabidopsis thaliana $R P P 8 / H R T$ family resistance gene, conferring resistance to Cucumber mosaic virus requires salicylic acid, ethylene and a novel signal transduction mechanism. Plant J. 32:655-667.

Takeda, A., Sugiyama, K., Nagano, H., Mori, M., Kaido, M., Mise, K. Tsuda, S., and Okuno, T. 2002. Identification of a novel RNA silencing suppressor, NSs protein of Tomato spotted wilt virus. FEBS (Fed. Eur. Biochem. Soc.) Lett. 532:75-79.

Thimm, O., Blasing, O., Gibon, Y., Nagel, A., Meyer, S., Kruger, P., Selbig, J., Muller, L. A., Rhee, S. Y. and Stitt, M. 2004. MAPMAN: a Userdriven tool to display genomics data sets onto diagrams of metabolic pathways and other biological processes. Plant J. 37:914-939.

Trinks, D., Rajeswaran, R., Shivaprasad, P. V., Akbergenov, R., Oakeley, E. J., Veluthambi, K., Hohn, T., and Pooggin, M. M. 2005. Suppression of RNA silencing by a geminivirus nuclear protein, AC2, correlates with transactivation of host genes. J. Virol. 79:2517-2527.

Ventelon-Debout, M., Delalande, F., Brizard, J. P., Diemer, H., Van Dorsselaer, A., and Brugidou, C. 2004. Proteome analysis of cultivarspecific deregulations of Oryza sativa indica and $O$. sativa japonica cellular suspensions undergoing Rice yellow mottle virus infection. Proteomics 4:216-225.

Wang, D., and Maule, A. J. 1995. Inhibition of host gene expression associated with plant virus replication. Science 267:229-231.

Whitham, S. A., Quan, S., Chang, H. S., Cooper, B., Estes, B., Zhu, T., Wang, X., and Hou, Y. M. 2003. Diverse RNA viruses elicit the expression of common sets of genes in susceptible Arabidopsis thaliana plants. Plant J. 33:271-283.

Zhu, S., Gao, F., Cao, X., Chen, M., Ye, G., Wei, C., and Li, Y. 2005. The Rice dwarf virus $\mathrm{P} 2$ protein interacts with ent-kaurene oxidases in vivo, leading to reduced biosynthesis of gibberellins and rice dwarf symptoms. Plant Physiol. 139:1935-1945.

Zhu, T., Chang, H. S., Schmeits, J., Gil, P., Shi, L., Budworth, P. R., Zou, G., Chen, C., and Wang, X. 2001. Gene expression microarrays: Improvements and applications towards agricultural gene discovery. JALA 6:95-98. 